\title{
Delineating Homology Generators in Graph Pyramids
}

\author{
Mabel Iglesias ${ }^{1,2}$, Adrian Ion $^{2}$, Walter G. Kropatsch ${ }^{2}$, and Edel B. García ${ }^{1, \star}$ \\ ${ }^{1}$ Pattern Recognition Department, Advanced Technologies Application Center, \\ Havana, Cuba \\ \{miglesias, egarcia\}@cenatav.co.cu \\ ${ }^{2}$ Pattern Recognition and Image Processing Group, \\ Faculty of Informatics, Vienna University of Technology, Austria \\ $\{$ ion, krw\}@prip. tuwien. ac.at
}

\begin{abstract}
Computation of homology generators using a graph pyramid can significantly increase performance, compared to the classical methods. First results in 2D exist and show the advantages of the method. Generators are computed on the upper level of a graph pyramid. Toplevel graphs may contain self loops and multiple edges, as a side product of the contraction process. Using straight lines to draw these edges would not show the full information: self loops disappear, parallel edges collapse. This paper presents a novel algorithm for correctly visualizing graph pyramids, including multiple edges and self loops which preserves the geometry and the topology of the original image. New insights about the top-down delineation of homology generators in graph pyramids are given.
\end{abstract}

Keywords: graphs pyramids, pyramid drawing, homology generators.

\section{Introduction}

Structural pattern recognition concerns the description and classification of objects, taking into account the relations between their individual parts and in some extent ignoring the changes of geometry caused by different transformations. We can break the geometric object into cells of dimension $0,1,2,3, \ldots$ corresponding to vertexes, edges, faces, volumes... After that, we can extract relevant topological information from the object. Algebraic topology has been applied to digital imagery in 12. As long as each cell that composes the object is homeomorphic to a topological ball, the homology of an image does not depend on its subdivision. Thus, the set of pixels can be considered as a cubical cell partition of the image space. Moreover, cubical homology theory has the ability to handle pixels or voxels directly without an artificial triangulation of every pixel or voxel. Computing homology generators means characterizing the holes in a

\footnotetext{
* Partially supported by the Austrian Science Fund under grants S9103-N13 and P18716-N13.
}

J. Ruiz-Shulcloper and W.G. Kropatsch (Eds.): CIARP 2008, LNCS 5197, pp. 576-584, 2008.

(C) Springer-Verlag Berlin Heidelberg 2008 
topological space. Some methods reduce the numbers of cells, thus the homology groups of an object composed of less cubes or simplices are computed. The method proposed in 3] also reduces the number of cells using a graph pyramid to efficiently compute the generators on top level. The authors claim that the generators computed stay on boundaries of the objects. In general, we observe that a set of generators for a group $H_{1}$ is a linear combination of 1 -holes. We want to visualize the generators at different levels of the pyramid, and understand the influence of the contraction process on the generators obtained on the top level. Top-level graphs may contain self loops and multiple edges, as a side product of the contraction process.

On the perspective of interactive visualization of graph pyramids, as far as we known, there is only one tool 4. This is oriented to facilitate the studies about the pyramid structure and improve the understanding of contraction process using the visualization options. This proposal uses straight lines which do not show the full information: self loops disappear, parallel edges collapse. On the other hand, there are applications where it is important to have a representation of a line drawing compressed without loss of its geometric structure and topology. In [5] a new rule based method on dual graph contraction is presented to built a graph pyramid in order to represent the line image. With this representation it is possible to detect features like loops and holes and to make use of relations like containment.

There is a graph drawing community interested in finding high quality of drawings in order to facilitate the visualization of complex relational networks. This means, for example, few edge bends in straight line drawings, orthogonal line segments for the edges, small display area, minimize crossings, good spatial and angular resolution, or recognizable symmetries. These methods find a distribution of nodes on the plane while we need to maintain a predefined position of nodes. In general, they do not deal with self loops and multiple edges as occurs in pyramids. The most prominent results can be seen in [6]. We present a novel algorithm for correctly visualizing graph pyramids preserving the geometry and the topology of the original image. In Section 2, basic notions related to graph pyramids, homology generators and their computation in a graph pyramid, are recalled. Sections 3 and 4 present the proposed algorithms, followed by experimental results. Section 5 concludes and gives an outlook of the future work.

\section{Recall}

\subsection{Graph Pyramids}

A graph pyramid $P$ is a stack of successively reduced graphs $P=\left\{G_{0}, \ldots, G_{h}\right\}$. Each level $G_{k}=\left(V_{k}, E_{k}\right), 1 \leq k \leq h$, is obtained by first contracting (contraction process) and then removing (simplification process) edges in the level $G_{k-1}$ below. Contracted edges of a level $G_{k-1}$ define trees called contraction kernels (CK) [7], whose vertices are merged to a single vertex in the level $G_{k}$ above. One vertex of each contraction kernel is called the surviving vertex and is considered to have been survived to the next level. Higher level vertices are related to the original input by equivalent contraction kernels (ECK) which, if applied, would achieve the 
same reduction in a single step. A path in a level $G_{k-1}$ is called a connecting path if it connects two (surviving) vertices $v, w$, and is made out of tree parts: a possible empty branch of the contraction kernel containing $v$, an edge called bridge that bridges the gap between two contraction kernels, and a possibly empty branch of the contraction kernel containing $w$. The base level bridge corresponding to an edge $e=(v, w)$ in a higher level $k$, is the bridge in the base level, connecting $E C K(v)$ and $E C K(w)$, and that has not been removed in any simplification up to the level $k$. The contraction process is controlled by the so called decimation parameters $\left(S_{k}, N_{k-1, k}\right)$, where $S_{k}=V_{k} \subset V_{k-1}$ is the set of surviving vertices and $N_{k-1, k}$ are the contraction kernels. $\left(S_{k}, N_{0, k}\right)$ denotes the surviving vertices and equivalent contraction kernels for contracting level 0 to level $k$.

Successive levels reduce the size of the data by a reduction factor $\lambda>1$. Each level represents a partition of the base level into connected subsets of pixels. The construction of a pyramid is iteratively local. On the base level (level 0) of a pyramid the cells represent single pixels and the neighborhood of the cells is defined by the 4-connectivity of the pixels. A cell on level $\mathrm{k}+1$ (parent) is a union of neighboring cells in level $\mathrm{k}$ (children). Every parent computes its values independently of other cells on the same level.

A level of a dual graph pyramid consists of a pair $\left(G_{k}, \overline{G_{k}}\right)$ of plane graphs $G_{k}$ and its geometric dual $\overline{G_{k}}$. The vertices of $G_{k}$ represent the cells on level $k$ and the edges of $G_{k}$ represent the neighborhood relations of the cells. The edges of $\overline{G_{k}}$ represent the borders of the cells on level $k$, including so called pseudo edges needed to represent neighborhood relations to a cell completely enclosed by another cell. Finally, the vertices of $\overline{G_{k}}$ represent junctions of border segments of $\overline{G_{k}}$. The sequence $\left(G_{k}, \overline{G_{k}}\right), 0 \leq k \leq h$ is called (dual) graph pyramid (DGP). For more details and formal definitions of the terms defined in this section see for example [7.

\subsection{Homology}

In this part, basic notions of homology theory are recalled. Interested readers can review algebraic topology books as 8,910.

In Fig. 1 a cell decomposition of an object $X$ is shown. A cell of dimension $p$ is called a $p$-cell (e.g. $f_{1}$ is a $2-$ cell and $a_{1}$ is a $1-$ cell). A $p$-chain is a lineal combination of $p$-cells of $X$ (e.g. $a_{2}+a_{7}+a_{4}$ is a 1 -chain). We define a set of homomorphisms $\partial_{p}$ in order to relate connectivity between two immediate dimensions. These applications $\partial_{p}$ describe the boundary of $p$-chain as $(p-1)$ chains.

The $p$-chain groups can be put into a sequence, in the following way:

$$
C_{n} \stackrel{\partial_{n}}{\longrightarrow} C_{n-1} \stackrel{\partial_{n-1}}{\longrightarrow} \cdots \stackrel{\partial_{1}}{\longrightarrow} C_{0} \stackrel{\partial_{0}}{\longrightarrow} 0
$$

which satisfy $\partial_{p} \partial_{p-1}(c)=0$ for any $p$-chain $c$. The boundary of a single $p-$ cell is defined as the sum of its incident $(p-1)$-cells. The boundary of a general $p$-chain is defined as the sum of the boundaries of each cell that appears in the chain (e.g. $\partial\left(f_{1}+f_{2}\right)=\partial\left(f_{1}\right)+\partial\left(f_{2}\right)=\left(a_{1}+a_{2}+a_{5}\right)+\left(a_{1}+a_{3}+a_{4}\right)=a_{2}+a_{3}+$ $\left.a_{4}+a_{5}\right)$. Since the chains are considered over $\mathbb{Z} / 2 \mathbb{Z}$ coefficients, any cell that appears twice vanishes. 
For each dimension $p$, the set of $p$ chains which have a null boundary are called $p$-cycles (e.g. $a_{1}+a_{3}+a_{4}$ is a 1-cycle). The set of $p$-chains which bound a $(p+1)$-chain are called $p$ boundaries (e.g. $a_{2}+a_{3}+a_{4}+a_{5}=\partial\left(f_{1}+\right.$ $\left.f_{2}\right)$ is a 1 -boundary). A $p$-hole is a $p$-cycle which is not a $p$-boundary (e.g $a_{2}+a_{6}+a_{7}$ is a 1 -dimensional hole).

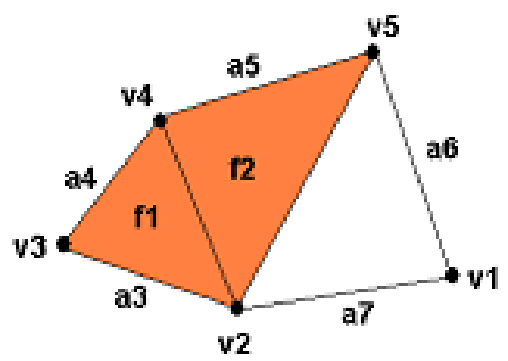

An equivalence relation which define that two cycles $z_{1}$ and $z_{2}$ are homologous if their difference is a boundary, (e.g $\left(a_{2}+a_{6}+a_{7}\right)-\left(a_{1}+a_{5}+a_{6}+a_{7}\right)=$ Fig. 1. Simplicial complex made of 1 connected component and containing one 1-dimensional hole $\left.\partial\left(f_{1}\right)\right)$ partitions the $p$-cycles in equivalence classes. Elements of the homology groups $H_{p}$ are equivalence classes and they surround the same holes.

A set $\left\{h_{1}, \cdots, h_{q} \mid h_{1} \in \mathcal{C}_{1}, \cdots, h_{q} \in \mathcal{C}_{q}\right\}$ [3] is called a set of generators for a group $H_{p}$ with $q$ independent equivalence classes.

\subsection{Computing Homology Generators in a Graph Pyramid}

The method in 3 follows the approach of reducing the number of cells of an object in order to compute homology. The idea is similar to [1112, but all simplifications computed during the reduction process are kept in using a pyramid (Fig. 2). In this way, homology generators are computed in the top level and are used to deduce generators of any level of the pyramid. The method can be summarized in three steps:

1. Starting from a segmented image, built a graph pyramid using contraction kernels of cells with the same label.

2. Homology generators are computed in the top level of the pyramid.

3. Deduce the homology generators of lowest level directly from the highest level using the notion of equivalent contraction kernel.

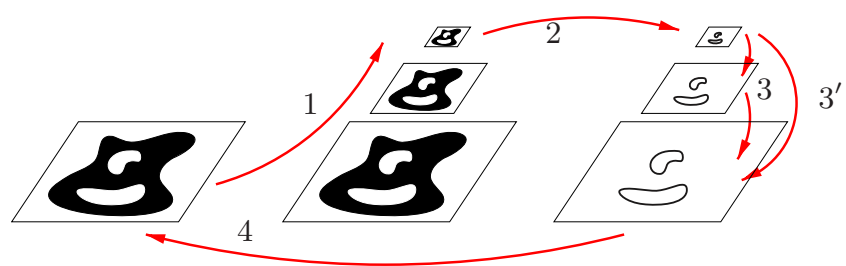

Fig. 2. Illustration of the method used in [3] for computing generators of homology groups using an image pyramid 
Visualization of homology generators computed on top level was only possible by down-projecting to the base level and showing the image itself. So far it was not possible to visualize them in the top level or any intermediate level.

\section{Visualizing a Graph Pyramid}

In the process of building a DGP the number of self loops and multiple edges is increasing, as some encode important information like inclusion and cannot be removed (see Fig. 3 c)). We propose a new method to correctly draw multiple edges and self loops maintaining the correspondences between surviving nodes.

The general problem is to draw a plane (multi)graph $G_{k}=\left(V_{k}, E_{k}\right)$ such that edges do not cross, assuming that the planar graph $G_{0}=\left(V_{0}, E_{0}\right)$ is defined on a square grid and $G_{k}$ has been constructed by dual graph contraction. The nodes inherit their positions in the plane and they should be kept in the same location. The general steps of the new algorithm are as follows:

1. Determine ECK corresponding to graph $G_{k}$, i.e. $\left(S_{k}, N_{0, k}\right)$.

2. For all the edges $e \in E_{k}$, find the corresponding bridge in $E_{0}$.

3. Calculate multiplicity of ECK-branches, i.e. count the number of bridges (of previous step) the branch is connected to.

4. Place interconnection squares where branches split or change direction.

5. Draw parallel lines according to the multiplicity count between all connected interconnection squares.

6. Connect lines inside interconnection squares without crossings.

The first step is to obtain the set of edges contracted from the base level graph that produced the actual graph. See Fig. 3 for an example. In general, this can be seen recursively as the ECK of the level k-2 plus the bridges of the edges in the CK of level k-1 $\left(N_{0,2}=N_{0,1} \cup \operatorname{BRIDGES}\left(N_{1,2}\right)\right)$. The edges obtained in step two plus the ones from the ECK obtained in the level before will be used as possible paths to draw connecting edges between surviving nodes. Note that each of these corresponding bridges from edges of graph $G_{k}$ is connecting two contracted trees, making the actual drawing connected. Dual graph contraction ensures the existence of connecting paths and that each connecting path contains exactly one bridge. In case of multiple connecting paths, the one that is not eliminated by the simplification process is used. The third step should start by the leafs of $N_{0, k}$ with multiplicity initialized in 0 and incremented by one for all incident bridges of step 2. All "interior" edges of $N_{0, k}$ sum the multiplicity of their sons plus, eventually, the number of incident bridges, see Fig. 3 d).

The set of edges that have multiplicity 0 are not useful for drawing so they are eliminated. Considering only the rest of the edges, for each "interior" node (non surviving node) check if there exists a branch split or direction change to place the interconnection squares. An interconnection square is placed only if it contains an adjacent edge with order greater than 1, see Fig. 3. e).

The interconnection squares where the edge orientation changes without splitting of branches have one side through which all lines enter and one side through 


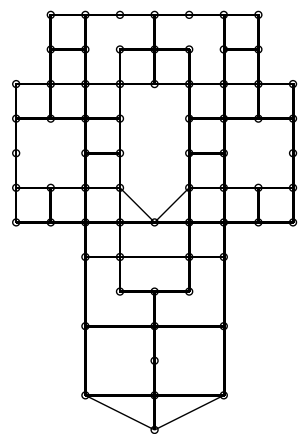

a) $G_{0}=\left(V_{0}, E_{0}\right)$

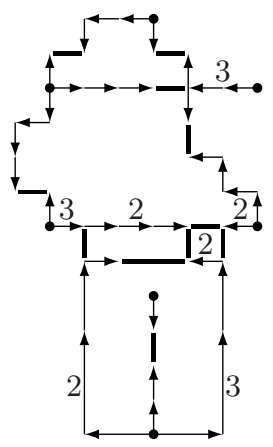

d)

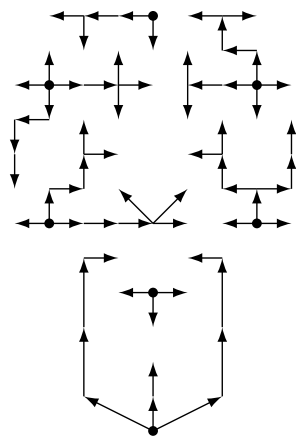

b) $N_{0,2}=E C K$

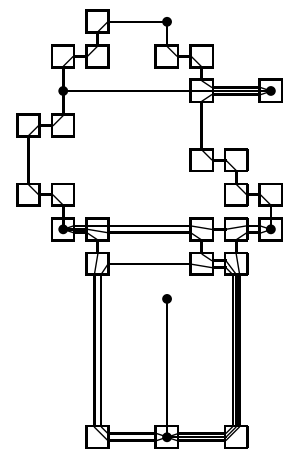

e)

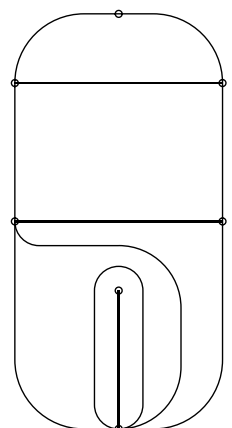

c) $G_{2}=C\left[G_{0},\left(S_{2}, N_{0,2}\right)\right]$

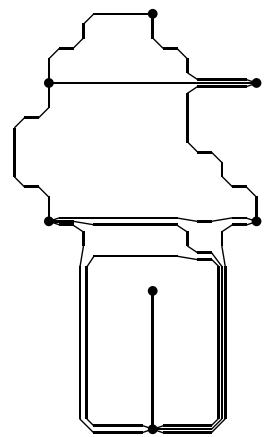

f)

Fig. 3. (a) base level; (b) ECK of level 2; (c) level 2; (d) connecting paths in $G_{0}$ of the edges in $G_{2}$ plus orders; (e) interconnection squares; (f) final drawing

which all lines exit. Hence, their order can be preserved without crossing. The interconnection squares at a branching correspond to a branching of a contraction tree (e.g a connected component of the ECK). Consequently there is only one side of the square through which the lines reach the tree's root. The multiplicity of this side is the sum of the multiplicity of all the other (3) sides. Furthermore, there is a strict order (i.e. clockwise) of the remaining three sides and the line bundles can be connected in the same order as shown, see Fig. 3 e). Finally, interconnection squares are not shown in the drawing, see Fig. 3 f).

\section{Experiments}

Until now, the visualization of homology generators, computed in a graph pyramid, was possible only by down-projecting them and then visualizing them in a refined resolution of the base level (image). Also, the homology generators are computed in the dual graphs $\overline{G_{k}}$, but the visualization was done in the image itself (see Figure 4). With the new method we are able to see all levels of a pyramid 

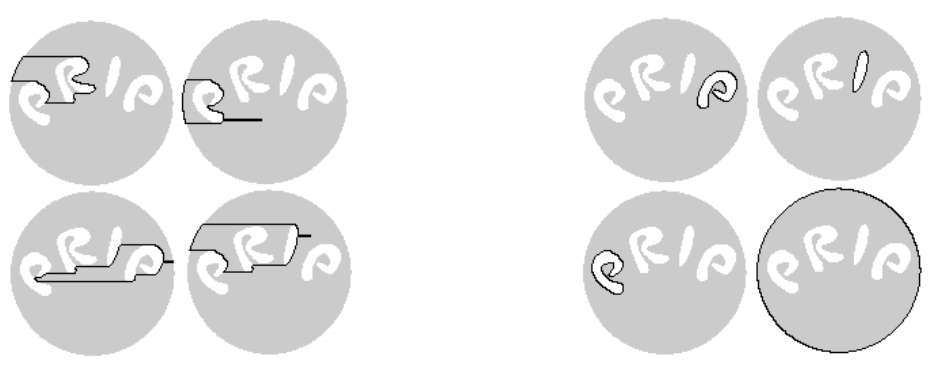

Fig. 4. Homology generators are computed in dual graphs but visualized on original image, left: without using pyramids, right: using pyramids and down-projecting them

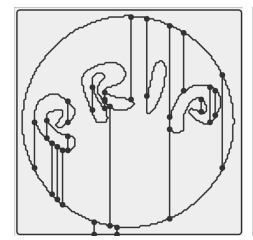

f)Level 30

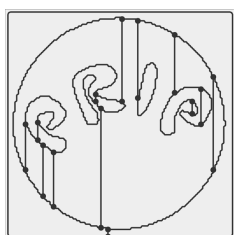

g)Level 31

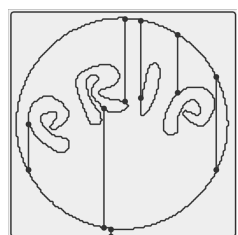

h)Level 32

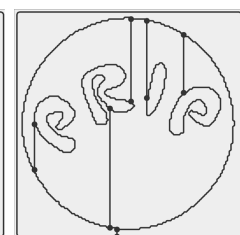

i)Level 33

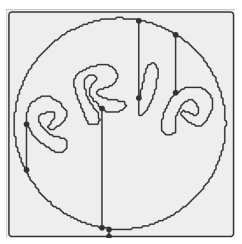

j)Level 34

Fig. 5. Graph Pyramid drawing

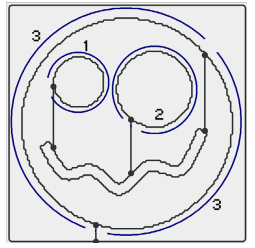

a) $G_{t}\left(I_{1}\right)$

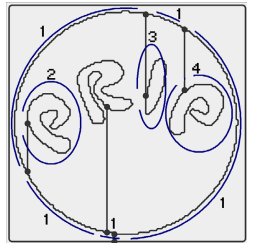

d) $G_{t}\left(I_{2}\right)$

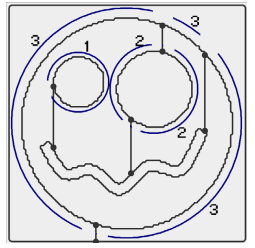

b) $G_{t-1}\left(I_{1}\right)$

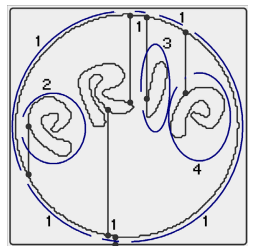

e) $G_{t-1}\left(I_{2}\right)$

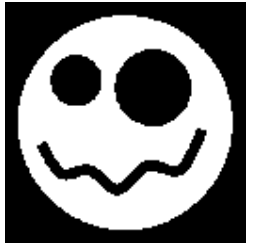

c) $\left.I_{1}\right)$

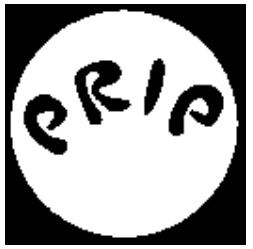

f) $I_{2}$

Fig. 6. First two top levels of pyramids showing generators for images $I_{1}$ and $I_{2}$. a), d) top level generators; b) and e) projected generators into the previous level.

and in particular the top levels where generators are computed (Fig. 4). Now, we are also able to see the generators's projections on the following levels, see Fig. 6. The figure shows the experiments using the images from [3. The image a-) has 3 
generators represented by numbers, the generator number 1 and 2 are self loops, and the third one is divided in two edges. The projection of this generators in the previous level(b), gives the generator 1 as a self loop but the generator 2 is now divided in two edges and the generator 3 is divided in 3 edges as well. The top level contains exactly a set of generators of the initial image. As shown, the graphs in those levels have self loops that now are nicely drawn and we can easily see the set of generators obtained by the method. The new method can correctly visualize all pyramid levels and any generators computed on them.

\section{Conclusion}

This paper presents a novel method for correctly visualize graph pyramids with multiple edges and self loops using not just straight lines, preserving the geometry and topology of the original image, and maintaining the correspondences between surviving nodes. Using straight lines to draw these edges would not show the full information: self loops disappear, parallel edges collapse. The usefulness of the method is shown, but not limited to, the context of homology generator computation using graph pyramids. We plan to extend this method for 3D combinatorial map pyramids, and apply it in the context of homology generator computation in $3 \mathrm{D}$.

\section{References}

1. Gonzalez, R., Real, P.: On the cohomology of 3d digital images. Discrete Appl. Math. 147(2-3), 245-263 (2005)

2. Boxer, L.: Digital products, wedges, and covering spaces. J. Math. Imaging Vis. 25(2), 159-171 (2006)

3. Peltier, S., Ion, A., Haxhimusa, Y., Kropatsch, W.G., Damiand, G.: Computing homology group generators of images using irregular graph pyramids. In: Escolano, F., Vento, M. (eds.) GbRPR. LNCS, vol. 4538, pp. 283-294. Springer, Heidelberg (2007)

4. Kerren, A.: Interactive visualization of graph pyramids. In: Graph Drawing, IBFI, Schloss Dagstuhl, Germany (2006)

5. Burge, M., Kropatsch, W.G.: A minimal line property preserving representation of line images. Computing 62(4), 355-368 (1999)

6. Duncan, C.A., Kobourov, S.G., Sander, G.: Graph drawing contest report. In: Hong, S.-H., Nishizeki, T., Quan, W. (eds.) GD 2007. LNCS, vol. 4875, pp. 395400. Springer, Heidelberg (2008)

7. Kropatsch, W.G.: Building irregular pyramids by dual graph contraction. IEEProc. Vision, Image and Signal Processing 142(6), 366-374 (1995)

8. Munkres, J.R.: Elements of algebraic topology. Perseus Books (1984)

9. Hatcher, A.: Algebraic Topology. Cambridge University Press, Cambridge (2002)

10. Zomorodian, A.: Topology for computing. Cambridge University Press, Cambridge (2005) 
11. Kaczynski, T., Mrozek, M., Slusarek, M.: Homology computation by reduction of chain complexes. Computers and Math. Appl. 34, 59-70 (1998)

12. Damiand, G., Peltier, S., Fuchs, L.: Computing homology for surfaces with generalized maps: Application to 3d images. In: Bebis, G., Boyle, R., Parvin, B., Koracin, D., Remagnino, P., Nefian, A., Meenakshisundaram, G., Pascucci, V., Zara, J., Molineros, J., Theisel, H., Malzbender, T. (eds.) ISVC 2006. LNCS, vol. 4292, pp. 235-244. Springer, Heidelberg (2006) 